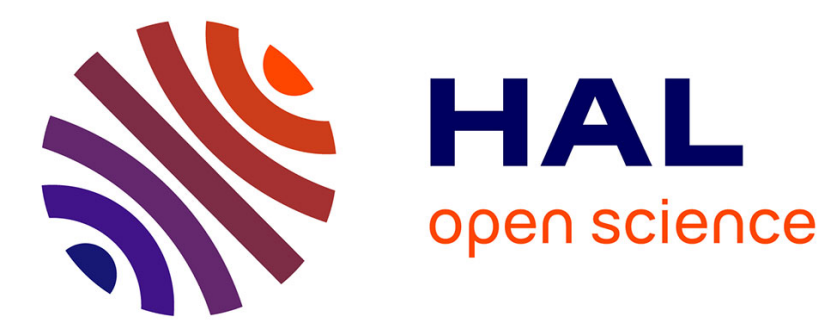

\title{
Polariton quantization modes and whispering gallery modes in a cylindrical microcavity
}

\author{
L.V. Kotova, P.G. Savvidis, L. Besombes, V.P. Kochereshko
}

\section{To cite this version:}

L.V. Kotova, P.G. Savvidis, L. Besombes, V.P. Kochereshko. Polariton quantization modes and whispering gallery modes in a cylindrical microcavity. Journal of Luminescence, 2021, 238, pp.118220. 10.1016/j.jlumin.2021.118220 . hal-03280439

\section{HAL Id: hal-03280439 \\ https://hal.science/hal-03280439}

Submitted on 7 Jul 2021

HAL is a multi-disciplinary open access archive for the deposit and dissemination of scientific research documents, whether they are published or not. The documents may come from teaching and research institutions in France or abroad, or from public or private research centers.
L'archive ouverte pluridisciplinaire HAL, est destinée au dépôt et à la diffusion de documents scientifiques de niveau recherche, publiés ou non, émanant des établissements d'enseignement et de recherche français ou étrangers, des laboratoires publics ou privés. 


\title{
Polariton quantization modes and whispering gallery modes in a cylindrical microcavity
}

\author{
L.V. Kotova ${ }^{\text {a, }}$, P.G. Savvidis ${ }^{\text {b,c }}{ }^{\text {, L. Besombes }}{ }^{\text {d }}$, V.P. Kochereshko ${ }^{\text {a }}$ \\ "Ioffe Inctinte, St Petersburg, 194021, Russia \\ ' Westake University, 18 Shillongshan Rd, Hangehou, 310024, Zhejiang, China \\ "Westiake Instimute for Advanced Snuly, 18 Shilangshan Rd, Hanghou, 310024, Zheriang, China \\ A Instint Noel, CNRS, Averue des Martyrs BP 166, Pr-38042, Grenoble, Colex 9, France
}

\section{A R T I L E IN F O}

\section{Keywonds}

Semiconductors

Spectroscopy

Exciton

Polariton

Microcavity
A B S T R A C T

Polariton photoluminescence from cylindrical microcavitiea was atudied under optical excitation below and above a nonlinear regime threshold. Lines of lateral excitonic polsritons quantization were observed in the apectra In addition to quantisation lines with a amall orbital quantum number $m$, exciton-polsriton whispering gallery modes with large $m>10$ were oboerved in the cavities of relatively large dismeter. In addition to the apectral distribution of the polsriton sive quantived modes, it was possible to oboerve their wavefunctions' apatial distribution.

\section{Introduction}

Microcavities are widely used to manufacture vertical surfaceemitting lasers (VCSELs) [1]. VCSELs have several advantages over conventional end-face lasers.

1. Since VCSELs are emitted from the top surface, they can be checked during fabrication, even before they are split into separate devices. This significantly reduces the yield of unusable and reduces the cost of the product.

2. It is possible to create two-dimensional arrays of VCSELs, entire matrixes from lasers - laser screens.

3. The large output aperture of the VCSEL provides a narrower output beam angle and provides high communication efficiency with optical fibers.

4. The small size of the active region, compared to edge-emitting lasers, reduces the VCSEL threshold current, which results in low power consumption [2]

Microcavities are also the basis for creating polariton lasers [3] These lasers do not use the reverse population effect to amplify light and stimulate emission, a phenomenon that underlies all lasers' operation and gave them their name. On the other hand, just like all lasers, a polariton laser is a source of coherent monochromatic light. The main advantage of polariton lasers over conventional lasers is their extremely low power consumption.

The process of creating an inverse population in conventional lasers is not energetically efflcient. It follows from Pauli's principle that the number of electrons that can simultaneously be at a given energy level is limited. As a result, conventional lasers consume energy to * throw * electrons to higher energy levels.

In a semiconductor polariton laser, electrons combine with holes to form excitons. Bxcitons are bosons and can be in an unlimited number at any energy level. An exciton, interacting with a photon, turns into a polariton, which emits a photon identical to the original one. The use of bosons can significantly reduce the threshold at which the laser can operate. As a result, polariton lasers consume hundreds of times less energy than traditional ones with the same radiation parameters. A polariton laser may be indispensable for creating quantum computers [4].

The most important element of any laser, both conventional and polariton, is the optical cavity. All lasers emit at frequencies corresponding to the eigenmodes of this cavity.

The microcavities with strong coupling were studied for several years [5] and the optical characterization of the lateral excited modes was observed. But, the regime of polariton laser $[6,7]$ was not enough studied.

In addition to the usual longitudinal modes, transverse optical modes

Corresponding suthor.

E-mail addres: kotova(iemail.ioffe.ru (L.V. Kotova). 
can also exist in cavities. Such modes are especially important for small resonators - microcavities.

Among such transverse modes, the most important are the so-called whispering gallery modes [8]. These standing waves experience total internal reflection from the resonator's sidewalls and are, as it were, "pressed s against them. As a result, such modes have a very high Q-factor [9] and can strongly affect the operation of both VCSEL and polariton lasers [10]. However, the extraction of radiation from such modes and the beam divergence is completely unsatisfactory [11].

In this work, we studied the emission spectra of the polariton lateral quantization modes in a cylindrical microcavity $14 \mu \mathrm{m}$ in diameter both in linear and nonlinear optical excitation regime at the temperature of 5 $\mathrm{K}$. It has been found that all lateral and whispering gallery modes are observed in the linear regime, and when switching to the lasing regime of a polariton laser, only one of the modes, as a rule, the lowest in energy is revealed.

\section{Experiment}

The studied samples were round mesa structures (mesas) with a diameter from 1 to $40 \mu \mathrm{m}$, made from microresonators, Fig. 1. The microresonator contains an $\mathrm{Al}_{0.25} \mathrm{Ga}_{0.75} \mathrm{As}, 5 \mathrm{\lambda} / 2$ - thick cavity surrounded by two Bragg mirrors, consisting of 35 pairs of AlAs and $\mathrm{Al}_{0.15} \mathrm{Ga}_{0.85} \mathrm{As}$ layers. The quality factor of the resonator was about 20.000 . This value was estimated from the half width of the reflection line (this is possible because the radiation leakage entirely determines the phonon mode's damping through the Bragg mirrors in the microcavity). Four GaAs quantum wells were placed inside the microcavity in the antinode of a standing electromagnetic wave to enhance the excitonphoton interaction. The wells' width was chosen to reach a resonance between the photon mode in the cavity and the ground state of the exciton in the wells, that is, the regime of strong exciton - photon coupling.

The photoluminescence (PL) spectra of these structures were registered at a temperature of $5 \mathrm{~K}$. For effective PL excitation, we used laser radiation with quantum energy $1.62 \mathrm{eV}(7652 \AA)$ ), which corresponds to the region above the stop band , but below the absorption band in Bragg mirrors. The spot diameter at the sample surface was about $2 \mu \mathrm{m}$. To record the $\mathrm{PL}$, a micro-photoluminescence setup was used. This setup consisted of a micro-objective, a monochromator, and a $\triangle C D$ camera.

Broad structureless emission band is observed at a sufflciently weal optical excitation (less than $0.03 \mathrm{~mW}$ ), in the PL spectrum of a mesa with a diameter of $14 \mu \mathrm{m}$, in the range from $8018 \AA$ to $8045 \AA$ (1.541-1.546 $\mathrm{eV}$ ). The absence of this band's structure is due to the effective population of the states of the entire polariton band under non-resonant photo excitation. With sufficiently large losses associated with exciton damping, standing waves cannot be formed in mesas of large diameter (tens of microns). Therefore, no structure is observed in the emission spectrum of polaritons.

The evolution of the PL spectrum with increasing optical excitation intensity is shown in flgures (Fig. 2 a-f). With an increase of the optical excitation power, when the a arrival of excitons begins to exceed thei losses due to damping, a set of narrow lines appears in the PL spectrum, associated with the exciton-polariton size quantization in the mesa plane (Fig. 2 a,b)

At a higher power of the optical excitation and a transition to the polariton laser mode, the intensity of the longest wavelength line in the spectrum begins to grow superlinearly, rapidly outstripping the growth of the intensity of the remaining lines. As a result, shortwave lines disappear, and only one long-wavelength line remains in the spectrum (Fig. 2 c). This regime, in fact, corresponds to Bose condensation of exciton-polaritons.

With a further increase in the excitation power, this line's width monotonically increases towards higher energies (Fig. $2 \mathrm{~d}-\mathrm{f}$ ). At low excitation power the full width at half maximum (FWHM) was about 0.1 $\mathrm{meV}$. At the highest intensity of optical excitation in our experiments, the FWHM of this line was $0.6 \mathrm{meV}$. Fig. 3 shows the dependences of PL intensity and FWHM of the lowest in the energy PL line. It can be seen in the inset to Fig. 3 that, at low excitation, the PL intensity linearly depends on the pump intensity $\left(I_{P L}=I_{e=}^{K}, K=1\right)$. This property is typical for excitonic PL when the carrier arrival and recombination are equal. When the excitation intensity reaches a threshold value of $0.03 \mathrm{~mW}$, the $\mathrm{PL}$ intensity grows superlinearly $K=2$ (when the excitation intensity changes by order of magnitude, the PL intensity increases by two orders of magnitude). This is the polariton laser regime. With a further increase in the pump intensity, we switch to the linear regime since other channels are included in the radiation process. The monotonic character of the broadening indicates that it is not associated with the contribution from neighboring lines to the width of this line. Simultaneously with the increase of width, the emission line shifts towards higher energies. In addition to this intense PL line, a weaker line is observed in the spectrum shifted by $\sim 0.5 \mathrm{meV}$ to higher energy. This line disappears from the spectrum at the highest excitation intensity.

Thanks to micro-PL, we were able to register the spatial distribution of polariton modes in the cavity and their spectral dependence (Fig. 4).

Fig. 4 on the left shows the photoluminescence spectrum. The horizontal scale represents the wavelengths (energies), and the vertical scale represents the spatial coordinate. Color represents the signal intensity in a logarithmic scale. Fig. 4 on the right shows the spatial distribution of the integral PL intensity. It is seen that the emission of long-wavelength modes of polariton quantization is concentrated closer to the center of the mesa and of short-wavelength modes to the edges. The shortest wavelength modes are * pressed * to the edge of the mesa. These are whispering gallery modec.

The line intensities in the PL spectrum in Fig. 4 on the left directly reflect the exciton-polariton wave function's spatial distribution on the size quantization levels. According to Formula 7, for low energy levels, the density of polaritons has a single maximum at the mesa center. For higher energy levels, there are several maxima and minima.

At excitation to the mesa's edge, the radiation focuses on the
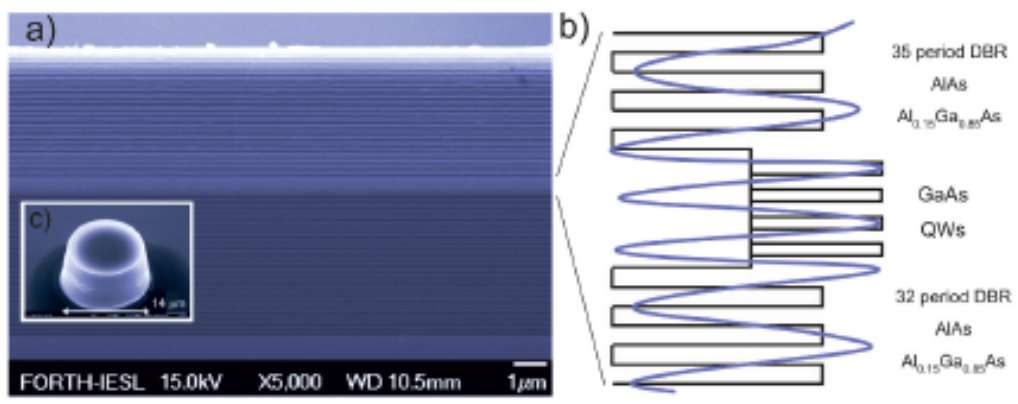

Fig. 1. The ample studied. a) Croos-aection of the asmple, b) band disgram. Insert (c) ahows the view of the meas. 

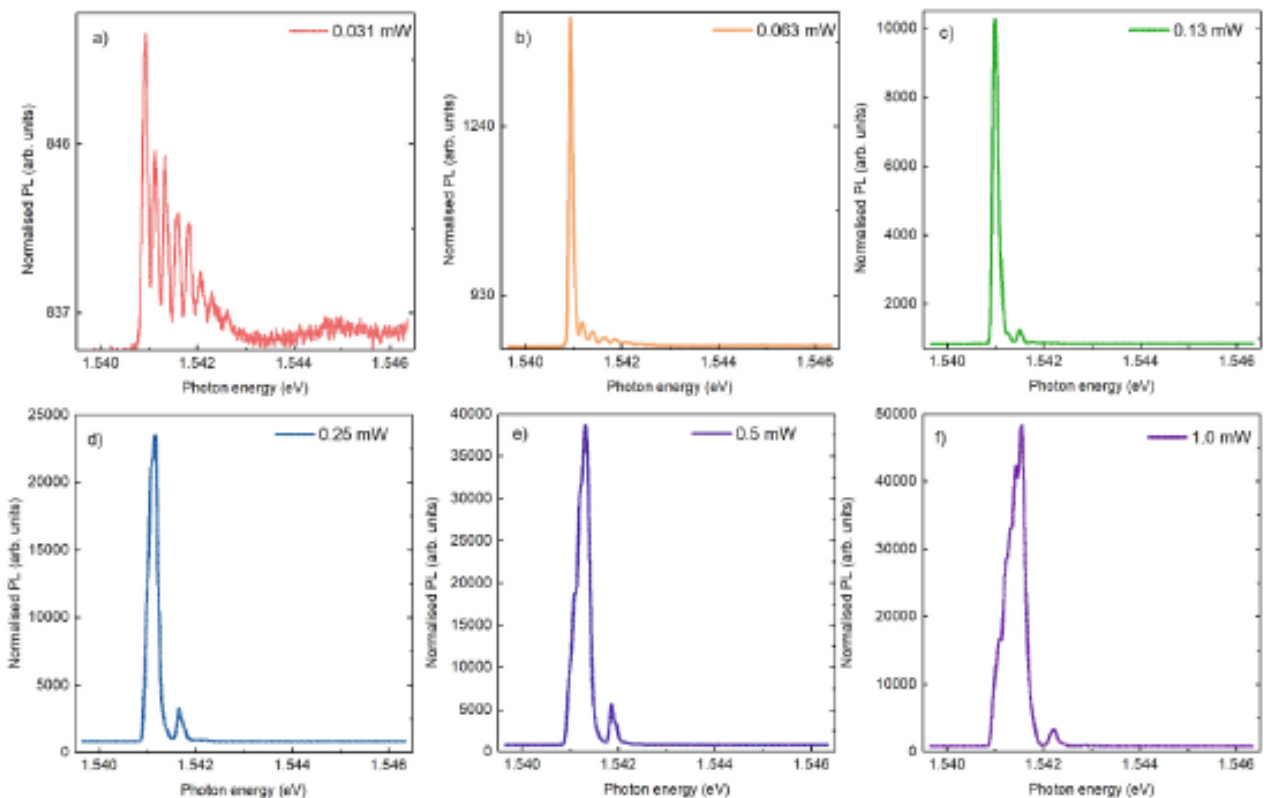

Fig. 2. a-f) Photoluminescence apectra taken from the mesa of $14 \mu \mathrm{m}$ as a function of the excitation power at $T=5 \mathrm{~K}$. Bxcitation denaity in (a) is $25 \mathrm{~W} / \mathrm{cm}^{2}$, in (b) is $50 \mathrm{~W} / \mathrm{cm}^{2}$, in (c) is $0.1 \mathrm{~kW} / \mathrm{cm}^{2}$, in (d) is $0.2 \mathrm{~kW} / \mathrm{cm}^{2}$, in (e) is $0.4 \mathrm{~kW} / \mathrm{cm}^{2}$, in (f) is $0.5 \mathrm{~kW} / \mathrm{cm}^{2}$. The cingle narrow longwave line in Fig. 2 (c) corresponda to the polariton lasing. The excitation powers are indicated in the insets to these figurea.

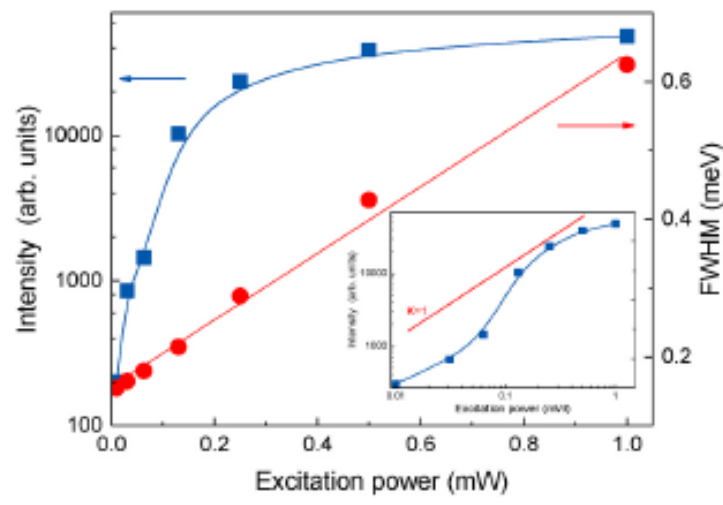

Fig. 3. Dependence of the PL intensity of the lowest line in the PL apectrum if Fig. 2 a and its FWHM on the excitation intensity. The inset thowe the aame dependence of the PL intensity $\left(I_{P L}=f\left(I_{e x r}\right)\right)$ in a double logarithmic ocale. An oblique atraight line corresponds to a linear relationahip $\left(I_{P L}=\alpha I_{\text {exr }}\right)$ between excitation and $\mathrm{PL}$

opposite point at the mesa's border (Fig. 5).

When the excitation point was changed at the edge of the mesa, the focal point also moved and occupied a position exactly opposite the excitation point

\section{Theory}

Under resonance conditions between the exciton and photon modes in a microcavity, excitons and photons cease to be independent of each other and form mixed states - polaritons. To describe such mixed states, it is necessary to solve jointly the equation of motion for the exciton polarization caused by the electromagnetic fleld and the equation for the electromagnetic field caused by the exciton polarization.

The equation for polarization $\mathbf{P}_{\text {exc }}$ associated with exciton excitation has the form [12]:

$\left[\hat{H}_{i j}(\mathbf{K})-h \omega \delta_{i j}\right] P_{i} \propto \sigma(\omega, \mathbf{K})=d_{i}(\mathbf{K}) \cdot d_{j}^{*}(\mathbf{K}) E_{j}(\omega, \mathbf{K})$

Here $d$ is the matrix element of the dipole moment of the exciton transition.

The equation of motion of the electromagnetic fleld under the action of exciton polarization

$$
\begin{gathered}
\Delta \mathbf{E}(\mathbf{r}, t)-\operatorname{grad}(\operatorname{div} \mathbf{E}(\mathbf{r}, t))=\frac{1}{c^{2}} \frac{\partial^{2}}{\partial r^{2}} \mathbf{D}(\mathbf{r}, t), \\
\mathbf{D}=c \mathbf{E}+4 \pi \mathbf{P}
\end{gathered}
$$

The system of differential Eqs. (1)-(2) must be solved jointly.

For a planar structure, the second term in (2) can be neglected. Although our structure is not completely planar, the diameter of the mesa is much larger than the thickness of the resonator, and the edge effects can be neglected. Then

$\Delta \mathbf{E}(\mathbf{r}, \omega)-k^{2} \mathbf{E}(\mathbf{r}, \omega)=4 \pi k_{0}{ }^{2} \mathbf{P}(\mathbf{r}, \omega)$

The equation for exciton polarization can also be rewritten as the inhomogeneous Helmholtz equation:

$\Delta \mathbf{P}(\mathbf{r}, \omega)-q^{2}(\omega) \mathbf{P}(\mathbf{r}, \omega)=d^{2} \mathbf{E}(\omega, \mathbf{r})$

Here: $q^{2}(\omega)=-\frac{2 M}{T}\left(\omega_{0}-\omega-i \Gamma\right), \omega_{0}$ is the exciton resonance frequency, $\Gamma$ is the exciton damping, $M$ is the exciton translational mass.

The condition for the solvability of the system of equations (3) - (4) leads to the dispersion equation [13]:

$\left(\omega_{\text {cts }}(k)-\omega-\Gamma\right)\left(\omega_{p t}(k)-\omega-i \gamma\right)=V^{2}$

Here: $h \omega_{c o x}(k)=h \omega_{c a x}^{0}+\frac{h^{2} k^{2}}{2 M}$ is the exciton energy, $h \omega_{p h}(k)=h \omega_{p h}^{0}+c h k$ 


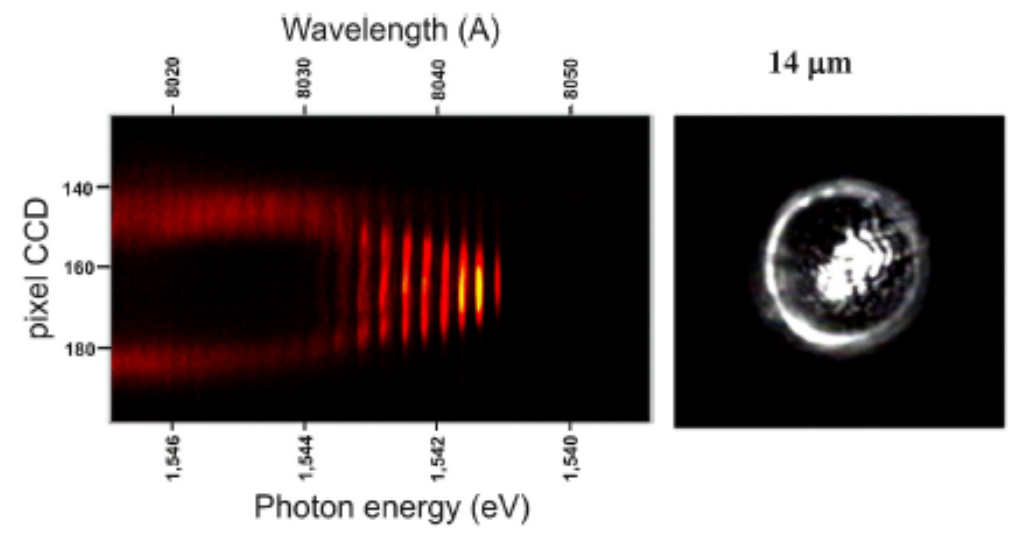

Fig. 4. Right - Integral photoluminescence of the meas with optical excitation to the center. Left - modes of lateral quantieation of polaritona and whispering gallery. Line intensities are ahown in color on a logarithrnic ocale.
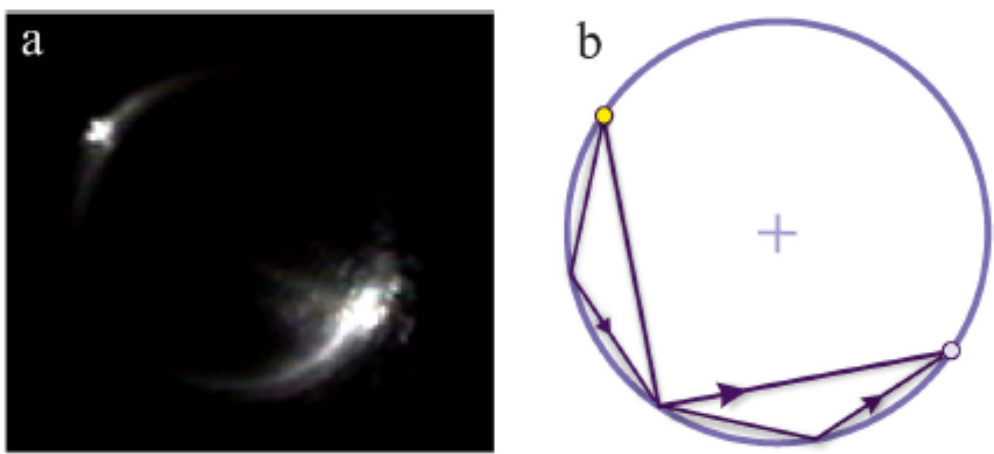

Fig. 5. a) Integrated photoluminescence of meas under optical excitation to the edge. b) Beam focuaing scheme.

is the photon mode energy, $c$ is the light velocity in a vacuum, $\gamma$ is the photon mode damping, $V$ is the parameter characterizing the interaction of the exciton and photon modes.

Under resonance conditions, the resonance frequencies of the exciton and photon are the same: $\omega_{\mathrm{exc}}^{0}=\omega_{\mathrm{ph}}^{0}=\omega_{0}$. Then:

$\omega_{1,2}=\omega_{0}+\frac{\omega_{\mathrm{M}}(k)}{2}-i \frac{(\Gamma+\gamma)}{2} \pm \Omega(k)$

$\Omega(k)=\frac{1}{2} \sqrt{\left[\omega_{M}(k)\right]^{2}+4 V^{2}-(\Gamma-\gamma)^{2}}$

Here:

$\omega_{M}(k)=c \sqrt{k_{p}{ }^{2}+k_{\perp}{ }^{2}}=c \sqrt{k_{p}{ }^{2}+\left(\frac{5 \pi}{L}\right)^{2}}$,

We assumed that the dispersion of the exciton is small at the scale of wave vectors of interest, $2 \Omega$ is Rabi splitting between exciton and photon modes.

The wave functions of polaritons in a cylindrical resonator are described as follows [13]:

$\Psi(\rho, \phi, z) \sim J_{m}(\rho) \sqrt{k_{\rho}^{2}+k_{\perp}^{2}} \sin \left(k_{\perp}\right) e^{ \pm i m \phi}$.

Here: $J_{m}(k \rho)$ are Bessel functions, $z$ is coordinate along the cylinder axis, $\phi$ is the angle in the resonator plane, $\rho$ is the coordinate along the resonator radius, $k_{\perp}$ is the wave vector along the axis $g, m$ is the numbe of the Bessel function, $n$ is the refractive index, $k_{0}=\omega / c$ is the wave vector of light in vacuum

To find the eigenmodes, it is necessary to set the boundary conditions. The general boundary condition requires that the linear combination of the function and its gradient along the normal to the surface be zero. For simplicity, we will assume that the eigenmodes correspond to solutions when $\Psi(\rho, \phi, \approx)=0$ on the surface. This should not greatly affect the result, but it will greatly simplify the calculations. The conditions on the quantum numbers $N, k_{\perp}$ and $m$ follow from the boundary conditions.

Quantization along the z-axis: $k_{\perp} L=\pi N, L=\frac{5}{2} \frac{1}{n}$ is the thickness of the active region of the resonator, $\left(n=3.5, \lambda_{0}=802.5 \mathrm{~nm}\right)$. Quantization in the plane of the structure obeys the condition: $k_{,} R=b_{l, m}, b_{l, m}$ are the roots of the Bessel functions: $J_{m}\left(b_{l m}\right)=0, l$ is the number of the root, $R$ is the radius of the mesa. We will talke into account only the first roots of each Bessel function $J_{\mathrm{m}}\left(b_{1, \mathrm{~m}}\right)$ since due to the presence of a mechanical (exciton) part of the polarization, polaritons quickly relax in energy.

From here we find all polariton modes of lateral quantization of polaritons in our resonator, they are indicated in Fig. 2 a. The result of the polariton dispersion curves calculation in the microcavity is shown in Fig. 6. In the calculation, we assumed the regime of the strong excitonphoton coupling $V \ngtr(\Gamma-\gamma)$. The calculation parameters $V$ and $\omega_{0}$ were chosen for better agreement between the calculation and experiment. The dots in Fig. 6 indicate the positions of the polariton size quantization 


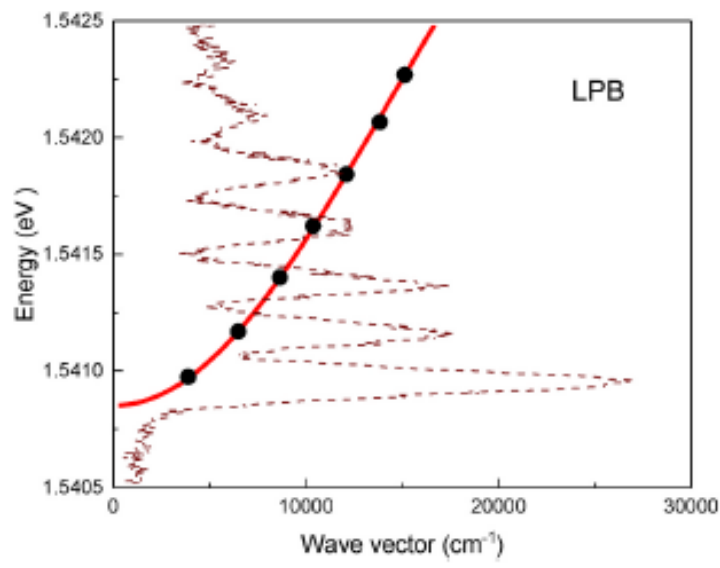

Fig. 6. Comparison of the PL apectrum ahown in Fign. $2 \mathrm{a}$ and Fig. 4 (left) and polsriton disperaion curves. The dependence of the polariton energy on ita wave vector (dispersion curve) was calculated under the sasumption of rero dampings $(\mathrm{T}=\gamma=0)$ in cylindrical coondinates. The closed circles ahow the quantised modes in a cylindrical resonator with a thickness $L=5 \lambda / 2=0.57 \mu$ and dismeter of $14 \mu \mathrm{m}$.

modes in our sample.

\section{The results and discussion}

At a weak optical excitation, a broad structureless emission band was observed in the photoluminescence spectra of cylindrical microcavities.

The absence of the structure of this band is due to rather large damping of exciton and photon modes because of leakage of radiation through the side surface. Due to the high losses, standing polariton waves cannot be formed, and in a structure of a sufficiently large size, a only wide PL band reveal. With an increase of the optical excitation power, modes of polariton lateral quantization appear in the spectrum. The number of these modes and the energy distance between them is determined by the dispersion of the polaritons in the cavity (Fig. 6).

Thanks to the use of micro-photoluminescence, it was possible to visualize the spectral and spatial distribution of all these modes. The spectral position of the PL peaks is determined by the quantization condition $k_{p}^{\mathrm{m}} R=b_{l, m}$, where $b_{l, m}$ are the roots of the Bessel functions. Fig. 4 shows that these peaks are modulated across the width of the mesa. This modulation is related to the factor $e^{ \pm \text {im } \phi}$ in the expression for the polariton wave function (Formula 7).

With an increase in the excitation intensity above a certain threshold, one or two of the lowest in energy polariton modes remain in the spectrum, and all other modes are suppressed. With a further increase in the optical excitation, the line remaining in the spectrum begins to broaden This broadening cannot be caused by the rise of excitonpolariton temperature. Indeed, in the mesa, polaritons are quantized in all three directions. Mesa is actually a quantum dot for polaritons. As a result, the polariton energy spectrum in the mesa is purely discrete, and, therefore, their energy levels cannot broaden with temperature increase. This circumstance is aggravated by the fact that polaritons are bosons, and one energy level can contain infinite polaritons.

We explain the broadening of the polariton laser line by the fact that not only excitons but also unbound electron-hole pairs are created under non-resonant optical excitation. The collision of excitons with free electrons and holes will lead to the broadening of the exciton PL line, as was observed in ordinary quantum wells [14]

An exciting feature of the whispering gallery modes is the focusing of radiation on the opposite edge of the mesa (Fig. 5). This effect can be qualitatively understood if we notice that all rays for modes with even numbers $m$ (Formula 7 ) converge at the point opposite to the excitation point. For rays with odd numbers, this is no longer the case; they are equally \& smeared s over the diameter. Then we get that the image of the radiation source located on the edge of the mesa will be focused to a point on the opposite edge of the mesa following the laws of the geometric optics.

This corresponds to the fact that the field amplitude in the resonator (Formula 4) is the same for arbitrary angles $\phi$ and $\phi+\pi$ all even $m$ from zero to $\infty$. Thus, if a fleld source is placed at a certain point with coordinates $(\rho, \phi)$, then its image will appear at a point with coordinates $(\rho$, $\phi+\pi$ ), that is, on the opposite edge of the mesa. The presence of the focusing confirms the fact that the radiation "pressed" to the edge of the mesa in Fig. 4 is associated namely with the modes of the whispering gallery.

\section{Conclusions}

In this work, we investigated the spectra of polariton photoluminescence from cylindrical microcavities under optical excitation below and above the threshold of transition to the nonlinear regime. Bright modes of exciton-polariton lateral quantization with a small orbital quantum number $m$ were observed in the spectra. In resonators of relatively large diameter exciton-polariton whispering gallery modes with large $m>10$ was observed.

Thanks to the use of micro-PL, in addition to the spectral distribution of the polariton size quantization modes, we were able to register the spatial dependence of the polariton wave function (Formula 7 ) in the cavity.

In the "polariton laser" regime, only one mode of polariton lateral quantization with the minimum energy * surviveds. The rest of the modes and modes of the whispering gallery, despite the higher quality factor, do not participate in the generation.

With a further increase in the excitation power, a broadening of the polariton laser emission line due to collisions of excitons with free carriers was observed.

\section{Authors statement}

L.V. Kotova: Investigation, Data curation, Visualization, Writing original draft, Writing - review \& editing; P.G. Savvidis: Resources, Investigation, Funding acquisition; L. Besombes: Conceptualization, Methodology, Investigation; V.P. Kochereshlo: Conceptualization, Data curation, Writing - review \&; editing, Project administration.

\section{Declaration of competing interest}

The authors declare that they have no known competing financial interests or personal relationships that could have appeared to influence the work reported in this paper.

\section{Acknowledgments}

LV.K. and V.P.K thank the RFBR grant No.19-02-00237 G.S.P. thanks the University Foundation of Westlake University and the program 2013R01002 supported by Leading Innovative and Bntrepreneur Team Introduction Program of Zhejiang.

\section{References}

[1] F. Koyama, S. Kinoshitn, K. Igr, Room temperature CW operation of GaAs vertical

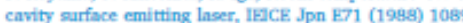

[2] V. Jayaraman, J. Jiang, B. Potsaid, G. Cole, J. Fujimoto, A. Cable, Design and

Performance of Broadly Tunable, Narrow Line-Width, High Reperition Pate Performance of Broadly Tunable, Narrow Line-Width, High Hepetition Hate pp. 99-109. 
[3] A. Imamoglu, R.J. Ram, S. Pau, Y. Yamamoto, Nonequilibrium condensates and lasers without inversion: exciton-polariton lasers, Phys. Rev. A 53 (1996) $4250-4253$.

[4] M.T. Hill, M.C. Gather, Advanoes in small lasers, Nat. Photonics 8 (2014) 908-918.

[5] R.L. Kaitouni, O. El Daif, A. Bans, M. Pichard, T. Paraiso, P. Lagan, I. Guillet,

F. Morier-Genoud, J.D. Ganiere, J.I. Staehli, V. Savona, B. Devenud, Engineering the spatial confinement of exciton polaritons in semiconductors, Phys. Rev. B 74 (2006), 155311.

[6] D. Bajoni, P. Senellart, A. Lemaltre, J. Bloch, Photon lasing in GaAs mierocavity: similarities with a polariton condensate, Phys. Rev. B 76 (2007), 201305.

[7] D. Tanese, H. Flayac, D. Solnyshkow, A. Amo, A. Lemaitre, E Galopin, R. Braive, P. Senellart, I. Sagnes, G. Malpuech, J. Bloch, Polariton condensation in solitoni: gap states in a one-dimensional periodic potential, Nat. Commun. 4 (2013) 1749.

[8] G.C. Righini, Y. Dumeige, P. Feron, M. Ferrary, G.N. Conti, D. Ristic, S. Sorid, Whispering gallery mode microresonators: fundamentals and applications, Riv. Nuovo Cimento 34 (2011) 435-489.
[9] X. Jiang, L. Shao, S.-X. Zhang, X. Yi, J. Wiersig, L. Wang, Q. Gong, M. Lontar, L. Yang, Y.$F$. Xiao, Chaos-assisted broodband momentum transformation in optical microresonators, Science 358 (2017) 344-347.

[10] E Gornik, Geometrical shaping of microlaser emission patterns, Science 280 (1998) 1544-1545.

[11] C.G.B. Garrett, W. Kniser, W.L. Bond, Stimulated emission into optical whispering modes of spheres, Phys. Fiev. 124 (1961) 1807-1809.

[12] E.I. Rashba, M.D. Sturge in: E.I. Ivchenloo (Ed.), * Excitonss in *Modern Problems in Solid State Physies Series, 2 ed., North-Holland Publishing Company, 1982.

[13] A.V. Kavoldin, J.J. Baumberg, G. Malpuech, F.P. Laussy (Eds.), Microcavity, Orford A.V. Kavolin, J.J. Baum bes
University Press, 2006.

[14] D.R. Yalkovlev, V.P. Kochereshlko, R.A. Suris, H. Schenk, W. Ossau, A. Wang. G. Landwehr, P.C.M. Christianen, J.C. Mnan, Combined exciton-cyclotron resonance in quantum well structures, Phys. Rev. Lett. 79 (1997) 3974-3977. 